\title{
Avaliação do conhecimento e percepção dos profissionais da estratégia de saúde da família sobre o uso de plantas medicinais e fitoterapia em Petrolina-PE, Brasil.
}

\author{
NASCIMENTO JÚNIOR, B.J."*; TÍNEL, L.O.2; SILVA, E.S.2; RODRIGUES, L.A.²; FREITAS, T.O.N.2; NUNES, \\ X.P.'; AMORIM, E.L.C. ${ }^{3}$ \\ 'Universidade Federal do Vale do São Francisco, UNIVASF. Colegiado de Farmácia. Av. José de Sá Maniçoba, \\ S/N, Centro, CEP: 56304-917, Petrolina, Pernambuco, Brasil.2Universidade Federal do Vale do São Francisco, \\ UNIVASF. Colegiado de Medicina. Av. José de Sá Maniçoba, S/N, Centro, CEP: 56304-917, Petrolina, \\ Pernambuco, Brasil. ${ }^{3}$ Universidade Federal de Pernambuco, UFPE. Pós-Graduação em Ciências Farmacêuticas. \\ Av. Prof. Moraes Rego, 1235, Cidade Universitária, Recife, Pernambuco, Brasil - CEP: 50670-901. "Autor para \\ correspondência: braz.jose@univasf.edu.br
}

\begin{abstract}
RESUMO: Este trabalho objetivou verificar se os profissionais de nível superior, ligados a Estratégia de Saúde da Família (ESF) do Município de Petrolina-PE percebem a importância, utilização e indicações de Plantas Medicinais e Fitoterápicos. Trata-se de um estudo transversal de caráter exploratório e descritivo, no qual participaram 96 profissionais de nível superior locados em Unidades da ESF. Os dados foram obtidos através de entrevista individual utilizando questionário semiestruturado. Para análise estatística, foi utilizado o teste Pearson Qui quadrado. As plantas medicinais mais citadas foram o Boldo (Plectranthus barbatus A.) e a Camomila (Matricaria recutita L.). Os fitoterápicos foram indicados apenas por $5(5,2 \%)$ médicos e os mais citados foram Guaco® e Maracugina ${ }^{\circledR} .36$ (37,5\%) dos profissionais acham que não estão preparados para repassar as informações sobre o uso de plantas medicinais e apenas $35(36,5 \%)$ se sentem preparados para prescrever fitoterápicos. Observa-se a necessidade de capacitação e motivação desses profissionais para a utilização correta e segura das Plantas Medicinais e Fitoterapia.
\end{abstract}

Palavras-Chave: plantas medicinais, fitoterapia, saúde da família.

\begin{abstract}
Knowledge assessment and perception of professionals of the family health strategy on the use of medicinal plants and phytotherapy in Petrolina, PE, Brazil. This study aimed to determine if the top-level professionals of the Strategy of Health of the Family, perceive the importance, use and indications of phytotherapy and Medicinal Plants. This is a cross-sectional, exploratory and descriptive study, with the participation 96 professionals serving in the family health strategy units. Data were collected through individual interviews in a semi-structured questionnaire. For statistical analysis, we used the Pearson Chi-square test. The most cited medicinal plants were the Boldo (Plectranthus barbatus A.) and Chamomile (Matricaria recutita L.). The phytotherapics were indicated only for $5(5.2 \%)$ physicians and the most cited were Guaco $₫$ and Maracugina $₫ .36$ (37.5\%) of the professionals think are not prepared to pass on the information about the use of medicinal plants and only $35(36.5 \%)$ feel prepared to prescribe phytotherapy. One observes the need for training and motivation of these professionals for the correct and safe use of Medicinal Plants and phytotherapy.
\end{abstract}

Keywords: medicinal plants, phytotherapy, family health.

\section{INTRODUÇÃO}

Os avanços científicos na área de medicamentos alopáticos, no século passado, proporcionaram o combate a alguns males que atingem a humanidade, como algumas doenças bacterianas, parasitárias e virais. Entretanto, $80 \%$ da população busca medidas terapêuticas alternativas devido à dificuldade de acesso ao atendimento primário à saúde, seja pela distancia dos grandes centros de referência, seja pela dificuldade e pela dificuldade de aquisição de medicamentos

Recebido para publicação em 20/02/2015

Aceito para publicação em 22/07/2015

$10.1590 / 1983-084 X / 15031$

Rev. Bras. PI. Med., Campinas, v.18, n.1, p.57-66, 2016. 
alopáticos devido a seu alto custo (Veiga Júnior, 2008).

Nesse sentido, as plantas medicinais e os fitoterápicos estão entre os principais recursos terapêuticos da Medicina Complementar e Alternativa e vêm sendo utilizados há muito tempo pela população brasileira nos seus cuidados com a saúde, na Medicina Tradicional/Popular ou nos programas públicos de fitoterapia no SUS, alguns com mais de 20 anos de existência. Entre as Práticas Integrativas e Complementares no SUS, as plantas medicinais e a fitoterapia são as mais presentes no Sistema, segundo diagnóstico do Ministério da Saúde, e a maioria das experiências ocorrem na Atenção Primária à Saúde. A Estratégia de Saúde da Família (ESF) e o Núcleo de Apoio à Saúde da Família (NASF) são fortalecidos ao se adotar e/ou estimular as plantas medicinais e a fitoterapia como uma de suas práticas de cuidado (Brasil, 2012).

A Resolução da Diretoria Colegiada da Agência Nacional de Vigilância Sanitária (RDC) $\mathrm{n}^{\circ} 26$ define fitoterápico como o produto obtido de matéria prima ativa vegetal, exceto substâncias isoladas, com finalidade profilática, curativa ou paliativa, incluindo medicamento fitoterápico e produto tradicional fitoterápico, podendo ser simples, quando o ativo é proveniente de uma única espécie vegetal medicinal, ou composto, quando o ativo é proveniente de mais de uma espécie vegetal e planta medicinal é a espécie vegetal, cultivada ou não, utilizada com propósitos terapêuticos (Brasil, 2014).

O uso de plantas medicinais passou a ser uma ferramenta importante dos profissionais de saúde, dos usuários, dos pesquisadores e dos gestores. O Ministério da Saúde criou a Política Nacional de Práticas Integrativas e Complementares no SUS. Posteriormente, também foram criados a Política Nacional de Plantas Medicinais e Fitoterápicos e o Programa Nacional de Plantas Medicinais e Fitoterápicos (Brasil, 2009a). Em 2008, foi publicada a Relação Nacional de Plantas Medicinais de Interesse do SUS (RENISUS), contendo 71 plantas (Brasil, 2009b).

Este artigo teve como objetivo verificar se os profissionais de nível superior, ligados à Estratégia de Saúde da Família do Município de Petrolina-PE percebem a importância, utilização e indicações de Plantas Medicinais e Fitoterápicos.

\section{MATERIAL E MÉTODOS}

A cidade de Petrolina está situada no estado de Pernambuco, distante de Recife, cerca de $721 \mathrm{Km}$. Possui clima semiárido, com vegetação predominante do tipo caatinga, sendo banhada pelo Rio São Francisco. A população do município é a quinta maior do estado, segundo o último censo, é de 293.962 habitantes (IBGE, 2010), dos quais 70\% residem na sede da cidade. Atualmente, existem 47 unidades UBS distribuídas pelos bairros do município e demais distritos que compõem a zona rural.

Essa pesquisa foi o resultado de um projeto de iniciação científica submetido ao Comitê de Ética e Deontologia em Estudos e Pesquisa (CEDEP UNIVASF), aprovado com registro № 0002/140613, de acordo com a resolução 466/2012 do Conselho Nacional de Saúde (Brasil, 2012) e a Declaração de Helsinque.

Trata-se de um estudo transversal de caráter exploratório e descritivo no qual participaram profissionais de nível superior locados em Unidades da Estratégia de Saúde da Família situadas na sede e interior do Município de Petrolina-PE. Os dados foram obtidos através de entrevista individual, usando-se um formulário específico contendo perguntas relativas ao conhecimento e utilização de plantas medicinais e fitoterápicos na atenção básica. $\mathrm{O}$ questionário utilizado foi o mesmo do estudo de Menezes et al. (2012), disponível na internet. As respostas foram anotadas no momento da entrevista, evitando-se falha de memória.

Todas as entrevistas foram feitas através de visitas às Unidades Básicas de Saúde, após os participantes assinarem o Termo de Consentimento Livre e Esclarecido (TCLE). Foi assegurado aos participantes a confiabilidade, sigilo e privacidade de sua identidade, utilizando-se códigos de identificação dos sujeitos. Além disso, foi assegurada a autonomia de recusar a participação e o direito de abandonar o estudo a qualquer momento.

Para o cálculo amostral, foi utilizado o Programa Estatístico Epi-info versão 6.0DOS. A seleção dos participantes se deu por amostragem não-probabilística, do tipo amostragem por Conveniência, ou seja, os participantes eram abordados e convidados a participar da pesquisa e recebiam uma numeração, sem a realização de sorteios (Martins \& Domingues, 2011). Para uma população de 155 profissionais cadastrados pela prefeitura de Petrolina, foi obtida uma amostra de $96(61,9 \%)$ profissionais, estimativa de erro igual a $5 \%$ e confiança de $95 \%$. Adotou-se para a análise estatística, o teste de Pearson qui quadrado, com nível de significância de $p<0,05$ (Hosmer \& Lemeshow, 2000).

\section{RESULTADOS E DISCUSSÃO}

Foram entrevistados 96 profissionais de nível superior que atuam na estratégia saúde da Família de Petrolina: $30(31,25 \%)$ Médicos, 36 (37,5\%) Enfermeiros e $30(31,25 \%)$ outros profissionais, como: $22(22,9 \%)$ Cirurgiões Dentistas,

Rev. Bras. PI. Med., Campinas, v.18, n.1, p.57-66, 2016. 
$6(6,3 \%)$ Farmacêuticos e $2(2,1 \%)$ Nutricionistas. Em relação à faixa etária, $50(52,1 \%)$ tinham menos de 30 anos. $70,8 \%$ eram do sexo feminino, 79,1 $\%$ tinham menos de 10 anos de formado, 18,7\% tinham entre 10 e 20 anos de formado e $59,4 \%$ tiveram a sua formação em uma instituição pública de ensino (Tabela 1).

Quando perguntados se os profissionais da saúde devem ter conhecimento sobre o uso e as indicações de fitoterápicos, observou-se que 95 (99\%) do total: $29(30,2 \%)$ médicos, $36(37,5 \%)$ enfermeiros, 22 (22,9\%) Cirurgiões Dentistas, 6 $(6,3 \%)$ Farmacêuticos e 2 (2,1\%) Nutricionistas responderam que sim. Não teve significância estatística $(p=0,695)$ entre os grupos. O resultado foi semelhante ao encontrado por Menezes et al. (2012), que foi de $100 \%$. Porém foi divergente com Dutra (2009), no qual encontrou realidades diferentes em relação às diversas classes profissionais: 100\% dos fisioterapeutas, farmacêuticos e cirurgiões dentistas; $86 \%$ dos técnicos em enfermagem, $65 \%$ dos enfermeiros e apenas $17 \%$ dos médicos são a favor do uso e indicações de fitoterápicos.

Quando questionados se sabem a diferença correta entre fitoterápicos e homeopáticos, observouse que $64(66,7 \%)$ do total: $13(13,5 \%)$ médicos, $28(29,2 \%)$ enfermeiros, $18(19,8 \%)$ Cirurgiões Dentistas, $3(3,1 \%)$ e $2(2,1 \%)$ responderam que não (Figura 1). Os resultados foram estatisticamente significantes quando cruzada com a variável profissão $(p=0,01)$ e com a variável Instituição de graduação $(p=0,028)$. Isso pode indicar a necessidade urgente de capacitação desses profissionais quanto ao uso dessa terapêutica complementar. Os profissionais que tiveram sua graduação em instituição pública $(59,4 \%)$ demonstraram conhecer mais a definição $(25 \%)$ que os graduados em instituição privada $(40,6 \%)$ que responderam a questão $(8,3 \%)$. Esses resultados podem indicar que os profissionais formados em instituição pública tiveram uma melhor formação na área de fitoterapia e homeopatia que os graduados em instituição privada, no grupo estudado. Percebe-se com o resultado que, o grupo dos médicos demonstrou um maior conhecimento nessa questão, fato que pode ser explicado pela formação do médico que é mais direcionada para prescrição de medicamentos que os outros profissionais da saúde. Outro fato que chamou a atenção no resultado dessa questão foi que os $3(3,1 \%)$ Farmacêuticos responderam que não sabiam diferenciar corretamente fitoterápicos de homeopáticos. Isso é preocupante, pois esse conhecimento é requerido como atribuição do profissional (Brasil, 2014). O resultado foi semelhante ao de Menezes et al.(2012), que encontraram $58(70,7 \%)$ dos profissionais de saúde sem o conhecimento diferencial entre os dois medicamentos.

Constatou-se que $87(90,6 \%)$ do total: $21(21,9 \%)$ médicos, $36(37,5 \%)$ enfermeiros, 22 $(22,9 \%)$ dentistas, $6(6,3 \%)$ farmacêuticos e 2 $(2,1 \%)$ nutricionistas responderam que toda equipe deve ter conhecimento sobre o uso de plantas medicinais (Figura 2). Nessa questão, o resultado foi estatisticamente significante $(p=0,0001)$, quando se relacionou com a profissão dos entrevistados. Desses, 9 (9,4\%) médicos não responderam que toda equipe deve conhecer as plantas medicinais, podendo indicar um pensamento individualista desse profissional na prescrição de medicamentos. Porém a RDC $\mathrm{N}^{\circ} 26$, afirma que não existe uma lista de fitoterápicos isentos de prescrição, mas que o uso desses medicamentos está mais ligado à indicação farmacológica que a prescrição exclusiva do médico (Brasil, 2014). Esse resultado corrobora com Saar \& Trevizan (2007), de que uma parte dos

TABELA 1.Respostas às perguntas de dados gerais por profissão de nível superior. Percepção dos profissionais da estratégia saúde da família sobre o tratamento com plantas medicinais e fitoterapia no município de Petrolina-PE, Brasil.

\begin{tabular}{lccccccc}
\hline Profissão & & Médico & Enfermeiro & Cir. Dentista & Farmacêutico & Nutricionista & $\%$ \\
\hline Número & & 30 & 36 & 22 & 6 & 2 & $\mathbf{1 0 0}$ \\
\hline \multirow{4}{*}{ Faixa Etária } & $20-30$ & 16 & 19 & 10 & 4 & 1 & $\mathbf{5 2 , 1}$ \\
& $31-40$ & 11 & 14 & 10 & 2 & 1 & $\mathbf{3 9 , 5}$ \\
& $41-50$ & 3 & 2 & 1 & 0 & 0 & $\mathbf{6 , 3}$ \\
\multirow{2}{*}{ Sexo } & $51-60$ & 0 & 1 & 1 & 0 & 0 & $\mathbf{2 , 1}$ \\
\hline \multirow{2}{*}{ Tempo de Formado } & $\mathrm{M}$ & 18 & 2 & 6 & 2 & 0 & $\mathbf{2 9 , 2}$ \\
(Anos) & $\mathrm{F}$ & 12 & 34 & 16 & 4 & 2 & $\mathbf{7 0 , 8}$ \\
\hline Instituição & 10 & 21 & 32 & 17 & 4 & 2 & $\mathbf{7 9 , 1}$ \\
de Ensino & +20 & 8 & 4 & 3 & 2 & 0 & $\mathbf{1 8 , 7}$ \\
\hline
\end{tabular}

Rev. Bras. PI. Med., Campinas, v.18, n.1, p.57-66, 2016. 


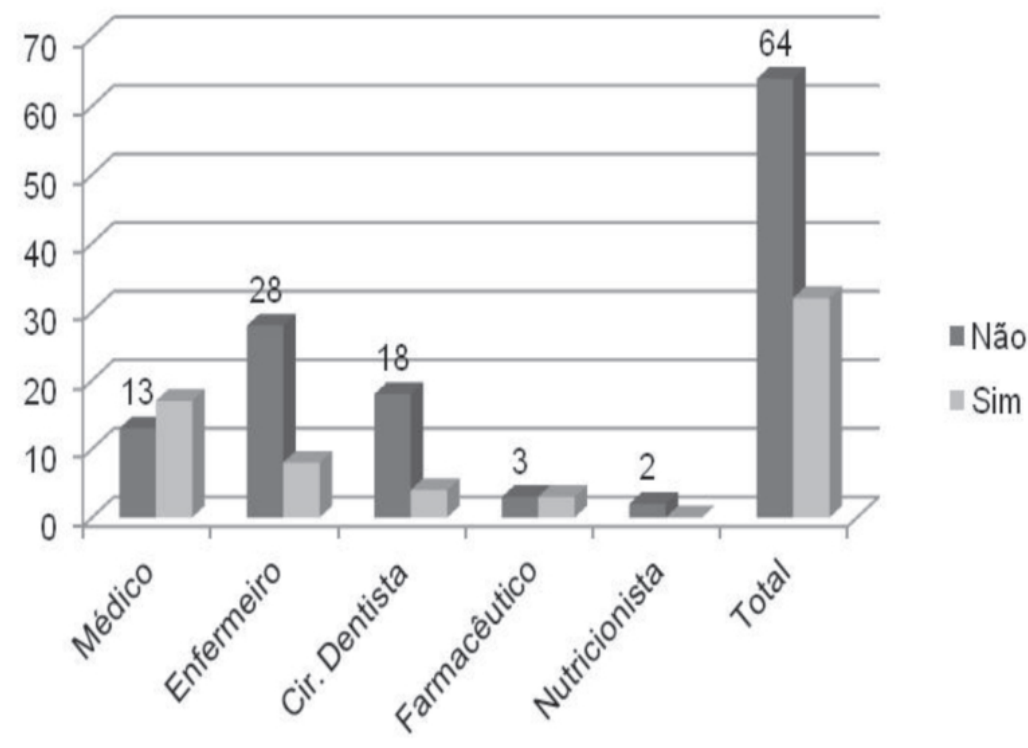

FIGURA 1. Respostas dos profissionais de nível superior que atuam em USF em Petrolina-PE, Brasil: sabe a diferença correta entre fitoterápicos e homeopáticos? Teste Pearson Qui-quadrado $(p=0,028)$

médicos é preconceituosa, resistente ao trabalho em equipe e centralizadora das ações. No estudo de Menezes et al. (2012), 79,3\% dos profissionais acham importante o conhecimento sobre o assunto por toda equipe.

Quando questionados se sabiam a definição de produtos fitoterápicos, observou-se que 48 $(50 \%)$ do total, $17(17,7 \%)$ médicos, $18(18,8 \%)$ enfermeiros, 9 (9,4\%) Cirurgiões Dentistas, 2 (2,1\%) Farmacêuticos e 2 Nutricionistas disseram que sim. Desses, 5 (5,2\%) médicos responderam de forma equivocada, pois afirmaram que Fitoterápicos são "plantas medicinais", "plantas medicinais para tratar doenças" ou "terapêutica com plantas medicinais". Como também, $3(3,1 \%)$ enfermeiros definiram de forma incorreta, que "fitoterápicos são plantas que agem na doença instalada", "utilização das plantas para solucionar problemas de saúde", "Extratos diluídos de elementos encontrados no ambiente natural". Em relação aos outros profissionais entrevistados, apenas 2 (2,1\%) Dentistas definiram incorretamente, "são medicamentos como chás

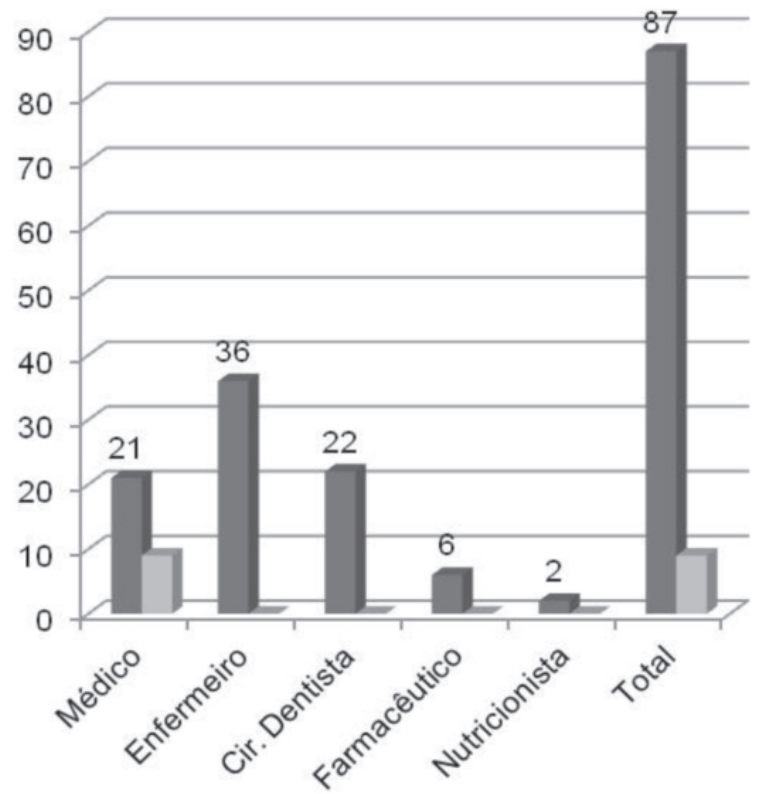

- Toda Equipe

" Algum Profissional

FIGURA 2. Respostas dos profissionais de nível superior que atuam em USF em Petrolina-PE, Brasil: Quais profissionais devem ter o conhecimento em Plantas Medicinais? Teste Pearson Qui-quadrado $(p=0,0001)$

Rev. Bras. PI. Med., Campinas, v.18, n.1, p.57-66, 2016. 
e infusões" ou "uso de plantas medicinais para tratamento ou prevenção de patologias". Percebese que alguns profissionais confundiram conceitos sobre plantas medicinais, fitoterápicos e até sobre homeopatia. Para Figueiredo \& Machado (2011) existe uma ideia equivocada entre os profissionais da saúde (gestores, médicos, cirurgiões dentistas, farmacêuticos e enfermeiros) e a comunidade em geral de que homeopatia é o mesmo que tratamento natural ou fitoterapia. Os resultados foram estatisticamente significantes quando cruzada a questão com a variável Instituição de graduação $(p=0,007)$. Isso pode indicar que os profissionais de instituição pública tiveram uma melhor formação na área de fitoterapia e plantas medicinais que os graduados em instituição privada, no grupo estudado. Esses resultados foram bem distintos de Menezes et al. (2012), onde 77 (93,9\%) dos profissionais souberam definir corretamente os medicamentos fitoterápicos.

Constatou-se que 35 (36,5\%) do total: 15 (15,6\%) médicos, 10 (10,4\%) enfermeiros, 8 (8,3\%) Cirurgiões dentistas, e 2 nutricionistas costumam prescrever esses medicamentos na ESF em que atua. Essa questão teve resultados estatisticamente significantes quando se cruzou com a variável profissão $(p=0,033)$ (Figura 3) e com a variável Faixa etária $(p=0033)$ (Figura 4). Os médicos do grupo estudado são os profissionais que mais prescrevem essas especialidades 15 (50\%) (Britto et al., 2007). Em relação à faixa etária, os profissionais com idades entre $20-30$ anos $(52,1 \%)$ prescrevem menos $(13,5 \%)$ fitoterápicos que os profissionais com idades entre $31-40$ anos (39,6\%) que prescrevem $(16,7 \%)$. Isso pode indicar que os profissionais mais velhos desse grupo, acreditam mais na eficácia dos fitoterápicos. As respostas foram conflitantes, pois nesse estudo apenas os médicos citaram nomes de fitoterápicos. Como é que alguns desses profissionais (não médicos) prescrevem essas especialidades farmacêuticas e não conhecem o nome dos medicamentos fitoterápicos? Esse fato pode ser em parte compreendido, porque os profissionais entrevistados não tinham formação e Fitoterapia e com isso, seus conhecimentos eram limitados. Fontenele et al. (2013) encontraram que $92,6 \%$ dos profissionais entrevistados citaram que seu conhecimento sobre fitoterapia é baseado principalmente na cultura popular, seja este isoladamente ou com interseção do conhecimento científico, havendo o reconhecimento de que seus conhecimentos específicos no assunto são limitados. No estudo de Menezes et al. (2012), $47,6 \%$ dos profissionais afirmaram que prescrevem esses produtos.

Quando questionados se fazem utilização pessoal das plantas medicinais, constatou-se que $47(49 \%)$ do total: $11(11,5 \%)$ médicos, $21(21,9 \%)$ enfermeiros, $12(12,5 \%)$ Cirurgiões dentistas, 2 Farmacêuticos $(2,1 \%)$ e 1 (1\%) Nutricionista responderam que sim. Os resultados não foram estatisticamente significantes $(p=0,414)$. Esses resultados são divergentes com os obtidos por Pires et al. (2014), que estudaram o uso de plantas medicinais em comunidade de Montes Claros - MG, eles encontraram uma prevalência na utilização de $75,5 \%$. Em outro artigo de Veiga Júnior (2008), a prevalência de utilização de plantas medicinais foi muito elevada pela população da região CentroNorte do Rio de Janeiro-RJ, alcançando $97,7 \%$ do total de entrevistados. Na pesquisa de Menezes et al. (2012), o percentual dos profissionais da saúde

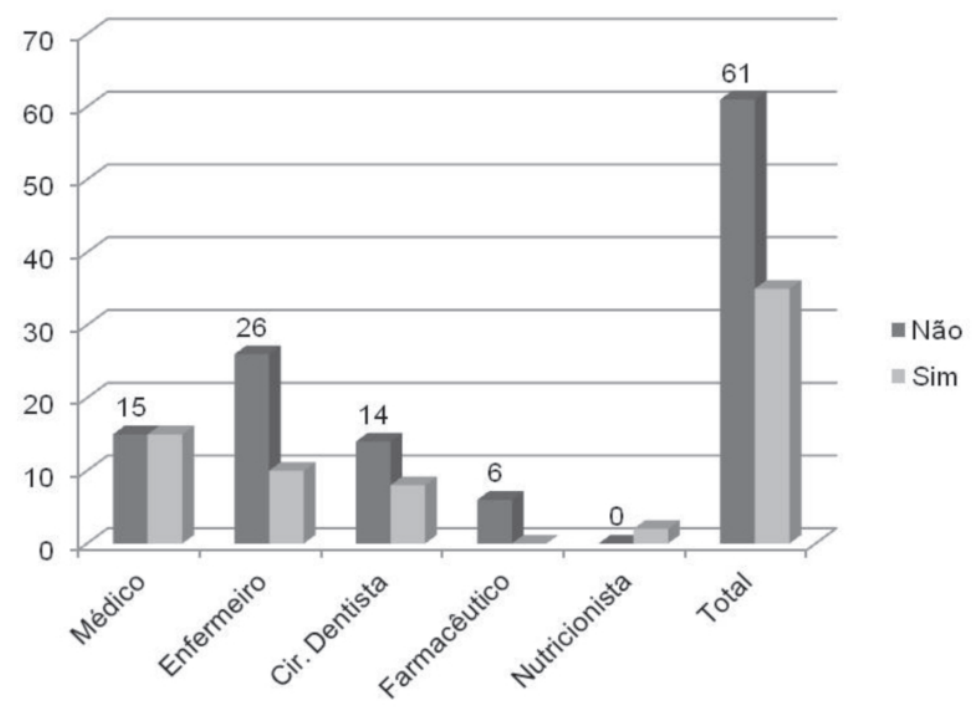

FIGURA 3. Respostas dos profissionais de nível superior que atuam em USF em Petrolina-PE, Brasil: Costuma prescrever fitoterápicos na ESF em que atua? Teste Pearson Qui-quadrado $(p=0,033)$

Rev. Bras. PI. Med., Campinas, v.18, n.1, p.57-66, 2016. 
que consomem planta medicinal foi de $62,2 \%$. Na pesquisa de Fontenele et al. (2013), a maioria dos profissionais de saúde já fez uso pessoal de plantas ou medicamentos fitoterápicos $(79,4 \%)$. Segundo relato dos entrevistados, esse uso teve como fonte de informações o contato com grupos culturais compreendendo parentes, amigos, vizinhos e os próprios usuários do SUS. Em estudo semelhante realizado com médicos no estado do Rio Grande do Sul, Rosa et al. (2011) trazem dados que corroboram esta realidade ( $77,8 \%$ de uso). Podemos suspeitar que o consumo de plantas medicinais esteja associado, em parte, aos hábitos culturais da região e ao poder aquisitivo da população, já que essas espécies costumam ser mais utilizadas pela população em geral que pelos profissionais da saúde. Percebe-se então, que apesar do profissional da saúde fazer uso de plantas medicinais em seu cotidiano, ele não se sente apto a prescrevê-las ou pela falta de crença nas práticas advindas da sabedoria popular (Brasil, 2006).

Observou-se que $60(62,5 \%)$ do total: $19(19,8 \%)$ médicos, 23 (24\%) enfermeiros, 14 $(14,6 \%)$ Cirurgiões dentistas e 4 farmacêuticos disseram não saber orientar seus pacientes sobre a forma de utilização de plantas medicinais. Não houve significância estatística para as repostas da questão $(p=0,489)$. O resultado foi semelhante ao da pesquisa de Menezes et al. (2012), que foi de $65,9 \%$. Esses dados estão de acordo com o estudo realizado por Petry \& Roman Júnior (2012), no qual afirmam que as plantas medicinais não estão disponíveis de maneira significativa no SUS, devido à falta de conhecimento dos profissionais da saúde.

Quando indagados se foram capacitados sobre a utilização de fitoterápicos durante sua formação, constatou-se que $67(69,8 \%)$ do total: 20 $(20,8 \%)$ médicos, 25 (26\%) enfermeiros, 18 (18,8\%) Cirurgiões dentistas, 2 (2,1\%) farmacêuticos e 2 $(2,1 \%)$ nutricionistas não receberam tal orientação. Não houve significância estatística nessa resposta $(p=0,178)$. O resultado encontrado foi bem menor que o de Menezes et al. (2012), em que $79,3 \%$ dos entrevistados não foram preparados na graduação. Apesar da fitoterapia ser reconhecida oficialmente em nosso país como terapêutica complementar, percebemos no presente estudo, que a maioria dos profissionais atuantes no Vale do São Francisco não recebeu instruções durante a graduação sobre fitoterapia e plantas medicinais, evidenciando as deficiências dos centros de formação, com ausência de disciplinas que abordem as práticas alternativas de saúde (Brasil, 2006). Para Rates et al. (2001) o problema da falta de capacitação seria resolvido se houvesse no currículo desses cursos, a disciplina de Farmacognosia, cuja base principal é o estudo dos aspectos farmacoterapêuticos de fitofármacos e fitoterápicos, visando ao estabelecimento de seu uso racional. Para Thiago \& Tesser (2011), esse conhecimento sobre as terapias complementares, deveria ser adquirido durante a formação, pois na pesquisa deles a maioria $(59,9 \%)$ dos entrevistados mostrou interesse em capacitações e todos concordaram que essas práticas deveriam ser abordadas na graduação.

Em relação às plantas medicinais utilizadas pelos 3 grupos estudados, os médicos citaram 9 espécies diferentes. As mais citadas foram: Camomila (Matricaria recutita L) (4 citações); Boldo (Peumus boldus M.) (3 citações) e Maracujá (Passiflora edulis S.) (3 citações). Os enfermeiros citaram 14 espécies diferentes e as mais prevalentes

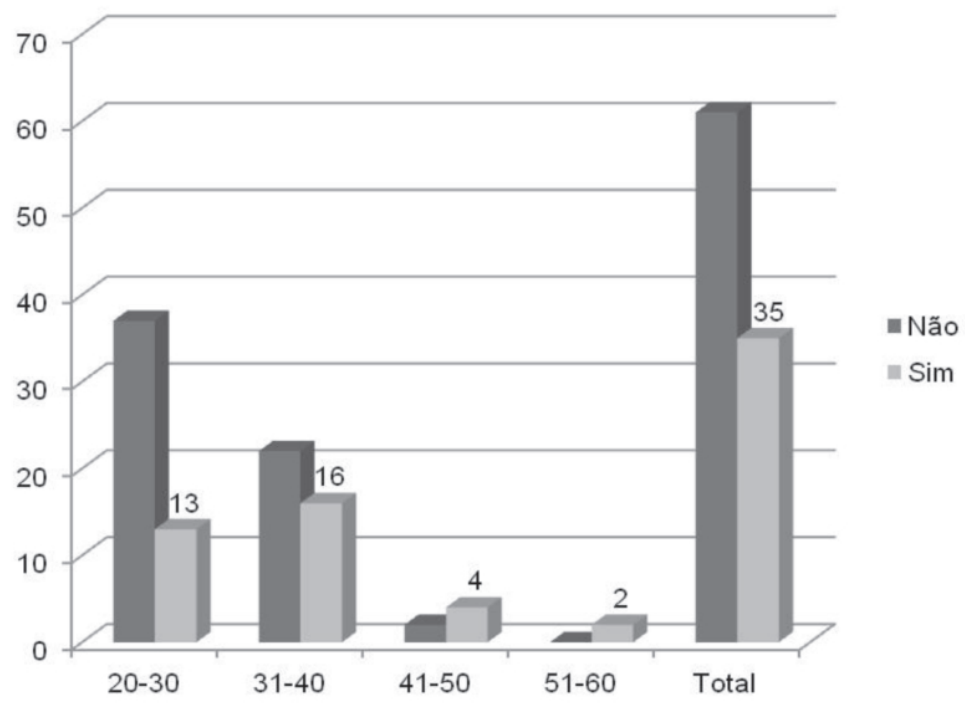

FIGURA 4. Respostas dos profissionais de nível superior, por faixa etária, que atuam em USF em Petrolina-PE, Brasil: Costuma prescrever fitoterápicos na ESF em que atua? Teste Pearson Qui-quadrado $(p=0,033)$

Rev. Bras. PI. Med., Campinas, v.18, n.1, p.57-66, 2016. 
TABELA 2. Plantas Medicinais prescritas pelos profissionais de nível superior com forma de preparo e indicações. Percepção dos profissionais da estratégia saúde da família sobre o tratamento com plantas medicinais e fitoterapia no município de Petrolina-PE, Brasil.

\begin{tabular}{|c|c|c|c|c|c|}
\hline Profissional & Espécies Vegetais & Parte Utilizada & Forma de Preparo & Indicação do Profissional & $\begin{array}{l}\mathrm{N}^{\circ} \text { de } \\
\text { Cita- } \\
\text { ções }\end{array}$ \\
\hline \multirow{9}{*}{ Médico } & Ameixa (Ximenia americana L) & Folhas & Infusão & Gastrite & 1 \\
\hline & Babosa (Aloe vera L.) & Folhas & $\begin{array}{l}\text { Aplicação local do gel } \\
\text { que sai das folhas }\end{array}$ & Cicatrizante, cosmético. & 2 \\
\hline & Berinjela (Solanum melongena L.) & Fruto & Cozido & Controle do colesterol, & 1 \\
\hline & Boldo (Plectranthus barbatus A. & Folhas & Infusão & Distúrbios Gastrointestinais & 3 \\
\hline & Camomila (Matricaria recutita L) & Flor & Decocção & Ansiedade & 4 \\
\hline & Erva doce (Pimpinella anisum $\mathrm{L}$ ) & Frutos & Infusão & $\begin{array}{c}\text { Expectorante e evita Flatu- } \\
\text { lência }\end{array}$ & 1 \\
\hline & Gengibre (Zingiber officinale R.) & Raiz & $\begin{array}{l}\text { Xarope, in natura e } \\
\text { decocção }\end{array}$ & $\begin{array}{l}\text { Rouquidão, expectorante, esti- } \\
\text { mulante gastrointestinal. }\end{array}$ & 1 \\
\hline & Maracujá (Passiflora edulis S.) & Polpa & Suco do Fruto & Ansiedade e Insônia & 3 \\
\hline & Quebra Pedra (Phyllanthus niruri L.) & $\begin{array}{l}\text { Caule, Folha e } \\
\text { Raiz }\end{array}$ & Decocção & Cálculo renal & 1 \\
\hline \multirow{14}{*}{ Enfermeiro } & Abacaxi (Ananas comosus L.) & Fruto, casca & Lambedor & Tosse e Gripe & 2 \\
\hline & Alho (Allium sativum L.) & Fruto & Pasta, in natura & $\begin{array}{c}\text { Diurético, laxante, cicatrizante, } \\
\text { bactericida. }\end{array}$ & 1 \\
\hline & Ameixa (Ximenia americana L) & Folhas & Infusão & Constipação & 1 \\
\hline & Aroeira (Schinus terebinthifolius R.) & Casca & Decocção & Prurido, anti-inflamatório & 3 \\
\hline & Berinjela (Solanum melongena L.) & Fruto & Cozido & Controle do colesterol, & 2 \\
\hline & Boldo (Plectranthus barbatus A. & Folhas & Infusão & Distúrbios Gastrointestinais & 9 \\
\hline & Camomila (Matricaria recutita $\mathrm{L}$ ) & Flor & Decocção & Ansiedade & 8 \\
\hline & Canela (Cinnamomum zeylanicum B.) & $\begin{array}{l}\text { Casca do } \\
\text { Caule }\end{array}$ & Decocção & Calmante, anti-inflamatório & 1 \\
\hline & Capim Santo (Cymbopogon citratus D.) & Folhas & Infusão & Calmante & 4 \\
\hline & Chuchu (Sechium edule J.) & Fruto, casca & $\begin{array}{l}\text { Decocção, triturado em } \\
\text { água. }\end{array}$ & Controle da Hipertensão & 3 \\
\hline & Cidreira (Lippia alba M.) & Folhas & Infusão & Calmante & 5 \\
\hline & Erva doce (Pimpinella anisum $\mathrm{L}$ ) & Frutos & Infusão & $\begin{array}{l}\text { Expectorante e evita Flatu- } \\
\text { lência }\end{array}$ & 1 \\
\hline & Marcela (Achyrocline satureoides L.) & Flores & Infusão & $\begin{array}{l}\text { Anti-inflamatório, analgésico, } \\
\text { calmante, antiespasmódico. }\end{array}$ & 1 \\
\hline & $\begin{array}{l}\text { Umburana de Cheiro (Amburana cearen- } \\
\text { ses D.) }\end{array}$ & $\begin{array}{l}\text { Sementes } \\
\text { pisadas }\end{array}$ & $\begin{array}{l}\text { Decocção do pó das } \\
\text { sementes assadas }\end{array}$ & $\begin{array}{l}\text { Problemas intestinais, bronco- } \\
\text { dilatador, anti-inflamatório. }\end{array}$ & 1 \\
\hline \multirow{4}{*}{$\begin{array}{l}\text { Cirurgião } \\
\text { Dentista }\end{array}$} & Aroeira (Schinus terebinthifolius R.) & Casca & Decocção & $\begin{array}{c}\text { Anti-inflamatório e antisséptico } \\
\text { bucal. }\end{array}$ & 2 \\
\hline & Camomila (Matricaria recutita $\mathrm{L}$ ) & Flor & Decocção & Controle do Biofilme Bucal & 2 \\
\hline & Gengibre (Zingiber officinale R.) & Raiz & $\begin{array}{l}\text { Xarope, in natura e } \\
\text { decocção }\end{array}$ & $\begin{array}{l}\text { Rouquidão, anti-inflamatório } \\
\text { para bochechos bucais. }\end{array}$ & 1 \\
\hline & Romã (Pumica granatum L) & Casca do fruto & Decocção & $\begin{array}{l}\text { Bochechos, anti-inflamatório } \\
\text { gengival. }\end{array}$ & 3 \\
\hline
\end{tabular}

\section{Farmacêutico Sem indicações de Plantas Medicinais ou Fitoterápicos}

\begin{tabular}{cllll}
\hline \multirow{2}{*}{ Nutricionista } & Camomila (Matricaria recutita L) & Flor & Decocção & $\begin{array}{l}\text { Calmante natural, atividade } \\
\text { antiespasmódica intestinal. }\end{array}$ \\
\cline { 2 - 5 } & Erva doce (Pimpinella anisum L) & Frutos & Infusão & Laxante e evita flatulência. \\
\end{tabular}


foram: Boldo (Plectranthus barbatus M.) (9 citações); Camomila (Matricaria recutita L) (8 citações) e Cidreira (Lippia alba M.) (5 citações). Os outros profissionais citaram 5 espécies vegetais diferentes e as mais citadas foram: Romã (Pumica granatum L) (3 citações); Camomila (Matricaria recutita L) (3 citações) e Aroeira (Schinus terebinthifolius R.) (2 citações) (Tabela 2).

Esses achados corroboram com os encontrados por Veiga Júnior (2008), que foram o Boldo (Plectranthus barbatus A.) e a Camomila (Matricaria recutita $L$ ), num estudo sobre o consumo de plantas medicinais na Região Centro-Norte do Rio de Janeiro. Em outro estudo, realizado por Brasileiro et al. (2008) sobre utilização de plantas medicinais em Governador Valadares, Minas Gerais, as duas espécies são bem citadas, porém a Erva Cidreira (Lippia alba M.) foi a mais prevalente do estudo com 986 citações, num total de 2454 entrevistas. No estudo de Menezes et al. (2012), a Hortelã (Mentha piperita L.) foi a planta mais prevalente nas prescrições dos profissionais, a Camomila (Matricaria recutita L.) aparece em segundo lugar e não há relato de uso do Boldo (Plectranthus barbatus A.) nesse estudo Albertasse et al. (2010) encontram a maior prevalência de citações $(9 / 198)$ para o uso do Boldo (Plectranthus barbatus A.). A Hortelã (Mentha piperita L.) foi a segunda planta mais citada (8/198). Em nosso estudo, a Hortelã (Mentha piperita L.), não foi citada pelos profissionais pesquisados.

Os Fitoterápicos só foram citados pelo grupo dos médicos, apenas 5 (16,6\%) profissionais
(Tabela 3). Os medicamentos citados nas entrevistas foram: Guaco® (Mikania glomerata S.) (4 citações); Maracugina $®$ (Passiflora alata A., Erythrina mulungu M. e Crataegus oxyacantha L.) (2 citações); Tensart® (Passiflora incarnata L.) (2 citações). Esses achados são semelhantes ao estudo de Silva et al. (2006), no qual o medicamento mais prescrito foi o Xarope Expectorante (composto por Guaco e Malvariço), que correspondeu a $63,8 \%$ de todos os fitoterápicos prescritos. O Maracujá (Passiflora edulis S.), apesar de ser produzido pelo laboratório de manipulação do Programa Farmácias Vivas de Maracanaú-CE é pouco prescrito pelos profissionais do local. Outro fato importante foi que um dos médicos entrevistados citou um medicamento que não é Fitoterápico, o Pantogar $\AA$, denotando desconhecimento sobre a definição desses medicamentos. Sobre o nosso estudo, Michiles (2004) ressalta a importância de recursos humanos capacitados, pois a utilização da Fitoterapia requer experiência técnica e/ou conhecimentos específicos, adquiridos em cursos de capacitação ou atualização constantes.

Não houve prescrição de fitoterápicos por profissionais não médicos, como Enfermeiros e outros profissionais nessa pesquisa. Alvim et al. (2006) discutem o uso de plantas medicinais e fitoterapia no cuidado de enfermagem, afirmando que o enfermeiro tende a reproduzir acriticamente o modelo biomédico, desconsiderando, por vezes, outras possibilidades de manifestação do saber sobre a saúde, como as advindas da sabedoria popular. Já Badke et al. (2011) afirmaram que é

TABELA 3. Medicamentos Fitoterápicos citados pelos Médicos. Percepção dos profissionais da estratégia saúde da família sobre o tratamento com plantas medicinais e fitoterapia no município de Petrolina-PE, Brasil.

\begin{tabular}{|c|c|c|c|}
\hline Fitoterápico & Planta & Indicações & $\begin{array}{c}\mathbf{N}^{\circ} \text { de } \\
\text { Citações }\end{array}$ \\
\hline Acheflan $^{\circledast}$ & Cordia verbenacea DC. & Analgésico e Anti-inflamatório & 1 \\
\hline Ansiopax ${ }^{\circledR}$ & Piper methysticum $\mathrm{F}$. & Estados de ansiedade, tensão e agitação & 1 \\
\hline Aplause ${ }^{\circledR}$ & Cimicifuga racemosa $\mathrm{L}$. & Alívio dos sintomas do climatério & 1 \\
\hline Calman ${ }^{\circledR}$ & $\begin{array}{l}\text { Passiflora incarnata L., Crataegus } \\
\text { oxyacantha L. e Salix alba L. }\end{array}$ & Ansiedade, distúrbios do sono na criança & 1 \\
\hline Fisioton ${ }^{\circledR}$ & Rhodiola rosea L. & Redução da fadiga e cansaço físico & 1 \\
\hline Guaco $^{\circledR}$ & Mikania glomerata S. & Expectorante, broncodilatador & 4 \\
\hline Maracugina $^{\circledR}$ & $\begin{array}{l}\text { Passiflora alata A., Erythrina mulungu } \\
\text { M. e Crataegus oxyacantha L. }\end{array}$ & Sedativo & 3 \\
\hline${ }^{*}$ Pantogar ${ }^{\circledR}$ & Não é Fitoterápico. & Queda de cabelo & 2 \\
\hline Sintocalmy ${ }^{\circledR}$ & Passiflora incarnata L. & $\begin{array}{l}\text { Estados de irritabilidade, tratamento de } \\
\text { insônia e desordens de ansiedade }\end{array}$ & 1 \\
\hline Tensart ${ }^{\circledR}$ & Passiflora Incarnata L. & $\begin{array}{l}\text { Estados de irritabilidade, tratamento de } \\
\text { insônia e desordens da ansiedade. }\end{array}$ & 2 \\
\hline
\end{tabular}

*Pantogar®, apesar de ter sido citado pelos médicos, não pode ser considerado Fitoterápico, pois não possui princípios ativos derivados de Plantas Medicinais.

Rev. Bras. PI. Med., Campinas, v.18, n.1, p.57-66, 2016. 
necessário um maior domínio desse saber pelos profissionais de enfermagem, pois este é um espaço do conhecimento popular que pode ser utilizado como um instrumento de proximidade, autonomia e de valorização da tradição dessas pessoas.

Em relação à prescrição de fitoterápicos por Cirurgiões Dentistas, Santos et al. (2009) ressaltam a importância da capacitação desses profissionais da área odontológica. Para Evangelista et al. (2013), os cirurgiões dentistas devem ser capacitados para o emprego das plantas medicinais com segurança, alicerçado nas evidências científicas. Adicionalmente, a aprovação da Política Nacional de Práticas Integrativas e Complementares (PNPIC) no Sistema Único de Saúde confirma a necessidade destes profissionais do SUS conhecerem e indicarem plantas medicinais e medicamentos fitoterápicos para suprirem as necessidades da população. Para Pontes et al. (2006), a falta de conhecimento e o pouco enfoque em terapias alternativas durante a formação acadêmica representam o principal motivo pelo qual a grande maioria dos profissionais de saúde não indicam medicamentos à base de Plantas medicinais.

\section{CONCLUSÕES}

Observa-se com esse estudo a necessidade de capacitação e motivação desses profissionais da saúde para a indicação das Plantas Medicinais e dos Medicamentos Fitoterápicos. Apesar de alguns profissionais afirmarem que prescrevem fitoterápicos, quando foi solicitado que os mesmos falassem nomes desses produtos, não se obtiveram respostas. O resultado foi conflitante e pode-se denotar que uma grande parte não está preparada para repassar informações sobre o uso de plantas medicinais ou prescrever Fitoterápicos para população, e isso, acaba acarretando um grande prejuízo para o serviço, pois essa Medicina Complementar e Alternativa é uma boa opção e de custo geralmente menor.

As escolas de formação deveriam incluir em seus currículos disciplinas, tais como a Fitoterapia, que aborde o tema e prepare os profissionais, bem como promovam cursos de reciclagem periódicos. Concomitantemente, a secretaria de saúde do município deveria investir na compra de fitofármacos, iniciar a implantação de laboratórios de manipulação de fitoterápicos e estimular a farmácia viva nas Unidades Básicas de Saúde.

Finalmente, o uso de Plantas medicinais e fitoterápicos tem como finalidade inserir outras opções terapêuticas alternativas e complementares, diminuir custos, retomar saberes tradicionais, conservar a biodiversidade, estimular o crescimento social, motivar as interações multisetoriais e interdisciplinares na educação comunitária em saúde e na participação coletiva, por todos esses benefícios deveria ser incluído e estimulado em todas as escolas de formação em saúde.

\section{AGRADECIMENTOS}

Agradecemos a Profa. Dra. Mônica Aparecida Tomé Pereira, coordenadora do Laboratório de Estatística Aplicada e Estudos Demográficos da Universidade Federal do Vale do São Francisco (UNIVASF), pelos cálculos estatísticos. Como também, aos programas PIBIC e Jovens Talentos para Ciência, pelo fornecimento de bolsas de Iniciação Científica para os alunos de graduação.

\section{REFERÊNCIAS}

ALBERTASSE, P.D.; et al., Plantas medicinais e seus usos na comunidade da Barra do Jucu, Vila Velha, ES. Revista Brasileira de Plantas Medicinais, v.12, n.3, p.250-260, 2010.

ALVIM, N.A.T., et al., O uso de plantas medicinais como recurso terapêutico: das influências da formação profissional às implicações éticas e legais de sua aplicabilidade como extensão da prática de cuidar realizada pela enfermeira. Revista Latino Americana de Enfermagem v.14, n.3, 2006.

BADKE, M.R.; ET AL., Plantas Medicinais: O Saber Sustentado na Prática do Cotidiano Popular, Revista da Escola Anna Nery, v. 15, n. 1, p.132-139, 2011.

BRASIL. Ministério da Saúde. Secretaria de Ciência, Tecnologia e Insumos Estratégicos. Departamento de Assistência Farmacêutica e Insumos Estratégicos. Programa Nacional de Plantas Medicinais e Fitoterápicos / Ministério da Saúde, Secretaria de Ciência, Tecnologia e Insumos Estratégicos, Departamento de Assistência Farmacêutica e Insumos Estratégicos. - Brasília: Ministério da Saúde, 2009a.

BRASIL. Ministério da Saúde. Direção de Administração e Finanças. Secretaria de Ciência, Tecnologia e Insumos Estratégicos. RENISUS - Relação Nacional de Plantas Medicinais de Interesse ao SUS. 2009b. 1p. Disponível em: http://portal.saude.gov.br/portal/arquivos/pdf/ RENISUS.pdf. Acesso em: 24 de setembro de 2014.

BRASIL. Ministério da Saúde. Resolução da Diretoria Colegiada - RDC N²6, de 13 de Maio de 2014. Dispõe sobre o registro de medicamentos fitoterápicos e o registro e a notificação de produtos tradicionais fitoterápicos. Agência Nacional de Vigilância Sanitária, 2014. Disponível em: http://bvsms.saude.gov.br/bvs/ saudelegis/anvisa/2014/rdc0026_13_05_2014.pdf. Acesso em 19 de novembro de 2014 .

BRASIL. Ministério da Saúde. Resolução n. 466/2012. Diretrizes e Normas Regulamentadoras de Pesquisa Envolvendo Seres Humanos. Brasília: Conselho Nacional de Saúde; 2012.

BRASIL. Ministério da Saúde. Secretaria de Atenção à Saúde. Departamento de Atenção Básica. Práticas integrativas e complementares: plantas medicinais e 
fitoterapia na Atenção Básica / Ministério da Saúde. Secretaria de Atenção à Saúde. Departamento de Atenção Básica. - Brasília: Ministério da Saúde, 2012.

BRASIL. Ministério da Saúde. Secretaria de Atenção à Saúde. Departamento de Atenção Básica. Política Nacional de Práticas Integrativas e Complementares no SUS - PNPIC-SUS / Ministério da Saúde, Secretaria de Atenção à Saúde, Departamento de Atenção Básica. - Brasília: Ministério da Saúde, 2006.

BRASILEIRO, B.G.; et al., Plantas medicinais utilizadas pela população atendida no "Programa de Saúde da Família”, Governador Valadares, MG, Brasil. Revista Brasileira de Ciências Farmacêuticas, v. 44, n. 4, p. 629-636, out./dez. 2008.

BRITTO, V.L.M.Q.; et al., Plantas medicinais e fitoterápicos no contexto da academia, governo e organizações da sociedade civil: exemplo de iniciativas populares no município de Uberlândia-MG, Revista de Educação Popular, v. 6, p.93-101. 2007.

DUTRA, M.G. Plantas Medicinais, Fitoterápicos e Saúde Pública: um diagnóstico situacional em Anápolis, Goiás. 2009. 112p. Dissertação (Mestrado - Área de Concentração em Sociedade, tecnologia e meio ambiente) - Centro universitário de Anápolis, UniEVANGÉLICA, Anápolis.

EVANGELISTA, S.S.; et al., Fitoterápicos na odontologia: estudo etnobotânico na cidade de Manaus. Revista Brasileira de Plantas Medicinais, v.15, n.4, 2013.

FIGUEIREDO, T.A.M.; MACHADO, V.L.T. Representações sociais da homeopatia: uma revisão de estudos produzidos no Estado do Espírito Santo, Revista Ciência \& saúde coletiva, v.16, s.1, p. 999-1005, 2011.

FONTENELE, R.P.; ETAL., Fitoterapia na Atenção Básica: olhares dos gestores e profissionais da Estratégia Saúde da Família de Teresina (PI), Brasil, Revista Ciências \& Saúde Coletiva, v.18, n.8, p. 2385-2394, 013.

HOSMER, D.W.; LEMESHOW, S. Applied Logistic Regression. 2.ed. New York: Wiley-Interscience Publication, 2000, 280p.

INSTITUTO BRASILEIRO DE GEOGRAFIA E ESTATÍSTICA. Sinopse do Censo Demográfico 2010. 1.ed. Rio de Janeiro: IBGE, 2011. 261p.

MARTINS, G.A.; DOMINGUES, O. Estatística Geral e Aplicada. 4.ed. São Paulo: Atlas. 2011. 680p.

MENEZES, V.A.; et al., Terapêutica com Plantas Medicinais: Percepção de Profissionais da Estratégia de Saúde da Família de um Município do Agreste
Pernambucano, Revista Odonto; v. 20, n. 39, p. 111122, 2012.

MICHILES, E. Diagnóstico situacional dos serviços de fitoterapia no Estado do Rio de Janeiro. Revista Brasileira de Farmacognosia, v.14, s. 0, p. 16-19. 2004.

PETRY, K., ROMAN JUNIOR, W.A. Viabilidade de implantação de fitoterápicos e plantas medicinais no Sistema Único de Saúde (SUS) do município de Três Passos/RS. Revista Brasileira de Farmacognosia, v. 93, n.1, p. 60-67, 2012.

PIRES, I.F.B., et al., Plantas Medicinais como opção Terapêutica em Comunidade de Montes Claros, Minas Gerais, Brasil. Revista Brasileira de Plantas Medicinais. v. 16, n. 2, s. 1, p.426-433, 2014.

PONTES, R.M.F.; et al., O uso da fitoterapia no cuidado de crianças atendidas em um centro de saúde do Distrito Federal. Revista Comunicação, Ciências e Saúde, v. 17, n. 2, p. 129-139, 2006.

RATES, S.M.K. Promoção do uso racional de fitoterápicos: uma abordagem no ensino de Farmacognosia. Revista Brasileira de Farmacognosia, v.11, n. 2, 2001.

ROSA, C.; ETAL., Representações e intenção de uso da fitoterapia na atenção básica à saúde. Revista Ciência \& Saúde Coletiva, v. 16, n. 1, p. 311-318, 2011.

SAAR, S.R.C.; TREVIZAN, MA. Papéis Profissionais de uma equipe de saúde: Visão de seus integrantes. Revista Latino Americana de Enfermagem, v. 15, n.1, 2007.

SANTOS, E.B.; et al., Estudo etnobotânico de plantas medicinais para problemas bucais no município de João Pessoa, Brasil. Revista Brasileira de Farmacognosia, v. 19, n. 1B, p. 321-324, 2009.

SILVA, M.I.G.; et al., Utilização de fitoterápicos nas unidades básicas de atenção à saúde da família no município de Maracanaú (CE). Revista Brasileira de Farmacognosia, v.16, n.4, p. 455-462, 2006.

THIAGO, S.C.S.; TESSER, C.D. Percepção de médicos e enfermeiros da Estratégia de Saúde da Família sobre terapias complementares, Revista de Saúde Pública, v.45, n.2, p. 249-257, 2011.

VEIGA JÚNIOR, V.F. Estudo do consumo de plantas medicinais na Região Centro-Norte do Estado do Rio de Janeiro: aceitação pelos profissionais de saúde e modo de uso pela população; Revista Brasileira de Farmacognosia, v. 18, n. 2, p. 308-313, 2008. 